\title{
Nevada de Reyes 2018 en Ávila y Segovia. Predicción, observación e impacto
}

\author{
Jesús Gordaliza Borrego ${ }^{1}$ (jgordalizab@aemet.es) \\ María Teresa García Gómez² (magarciag@aemet.es) \\ ${ }^{1}$ AEMET / Delegación Territorial en Castilla y León \\ ${ }^{2}$ AEMET / Dirección de Producción e Infraestructuras / Área de Técnicas y Aplicaciones de Predicción
}

\section{RESUMEN}

Los días 6 y 7 de enero de 2018 se registraron en las provincias de Ávila y Segovia nevadas muy significativas tanto en duración como en intensidad. Estas nevadas tuvieron un gran impacto en la población, especialmente en las comunicaciones por carretera añadido al hecho de una operación retorno de vacaciones de Navidad.

Se analizarán las herramientas de predicción, la predicción operativa, la observación, datos registrados e impacto en la población.

PALABRAS CLAVE: predicción; nevada; vigilancia; impacto; avisos.

\section{INTRODUCCIÓN}

En esta ponencia se pretende presentar un caso concreto de un episodio de fenómeno meteorológico adverso como es la nieve.

Este episodio de nieve se sitúa temporalmente en los días 6 y 7 de enero de 2018 y geográficamente afectó a todo el norte peninsular español y donde más impacto tuvo fue en las provincias de Ávila y Segovia, por lo excepcional del episodio y por su coincidencia con la festividad de Reyes con la operación retorno de Navidad por carretera.

El impacto principal fue el de numerosas carreteras impracticables o en malas condiciones de tránsito. Incluso la autopista AP-6 que une la Meseta y todo el noroeste de España con Madrid quedó colapsada y numerosos coches quedaron atrapados durante la noche de Reyes.

\section{PREDICCIÓN}

Presentamos cómo el Sistema Nacional de Predicción de AEMET predijo este episodio a través de sus predicciones y avisos especiales, así como de sus avisos por acumulación de nieve en 24 horas.

También se mostrarán diversos productos de los modelos numéricos de predicción que se utilizaron por los predictores operativos. 


\subsection{Medio plazo}

El 1 de enero, los escenarios de predicción por conjuntos (EPS) del ECMWF para el día 6 a las 12 UTC ya indicaban la alta probabilidad de la formación de una profunda borrasca, tanto en superficie como en niveles medios.

En la figura 1 se puede observar que aunque el EPS agrupa los 51 posibles escenarios en 6 agrupaciones, 5 de estas agrupaciones que son las más numerosas, apuntan a la formación de esta borrasca acompañada de precipitaciones en forma de nieve en el centro peninsular; el grupo 6 y menos numeroso sitúa la borrasca más al este de la Península con mucha menos precipitación en el centro.

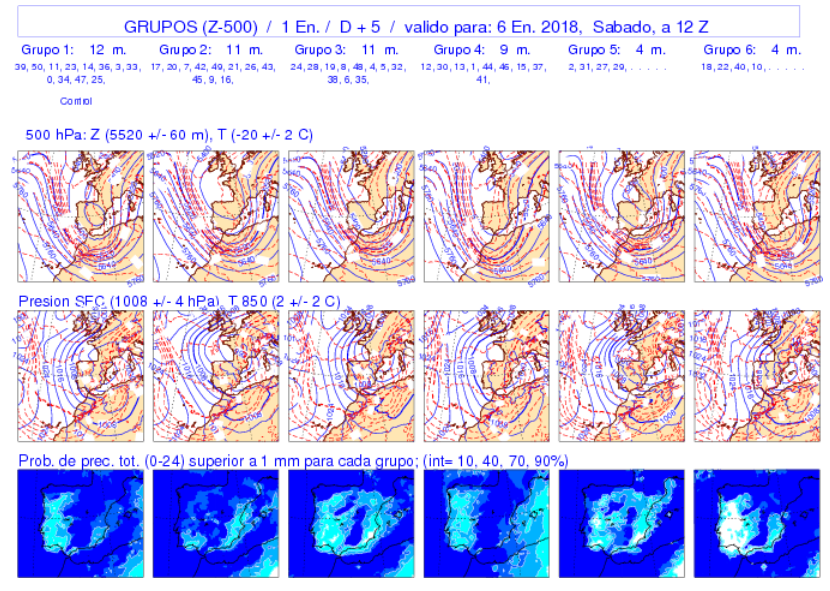

Figura 1. EPS del ECMWF del día 1 previsto para el día 6 a las 12 UTC.

Por tanto, a cinco días vista, era alta la probabilidad de un episodio significativo de nieve en el centro de la Península.

\subsection{Corto plazo}

La alta probabilidad de episodio de nevadas se mantuvo durante toda la semana.

El mismo día 6 por la mañana, había poca incertidumbre en las predicciones de los modelos. Así lo diagnosticó el Centro Nacional de Predicción y así lo pronosticaba el modelo ECMWF: formación de una borrasca en la Península con una marcada vaguada en altura y con una anomalía fría de $-30{ }^{\circ} \mathrm{C}$ (figuras de 2 a 5); lo que implicaba precipitaciones persistentes en forma de nieve en todo el centro y norte peninsular.

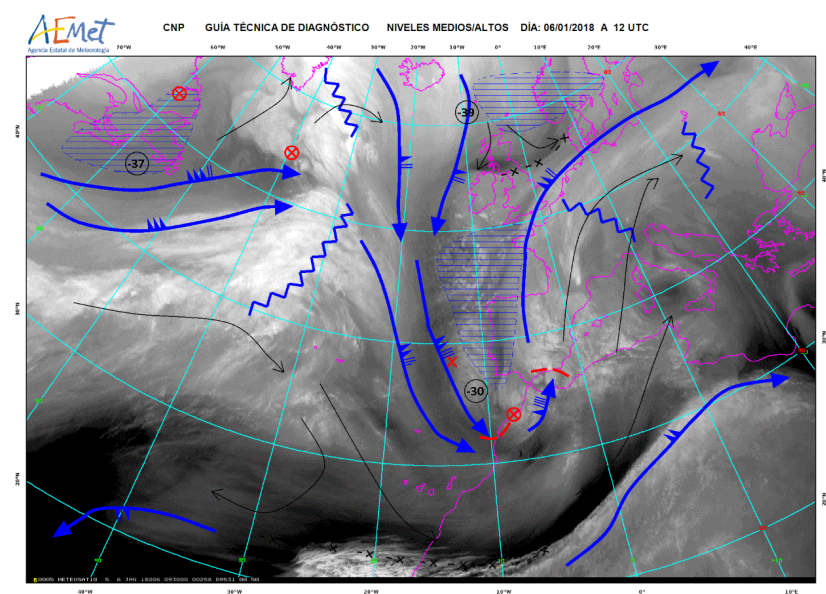

Figura 3. Diagnóstico en niveles medios/altos a las 12 UTC del día 6.

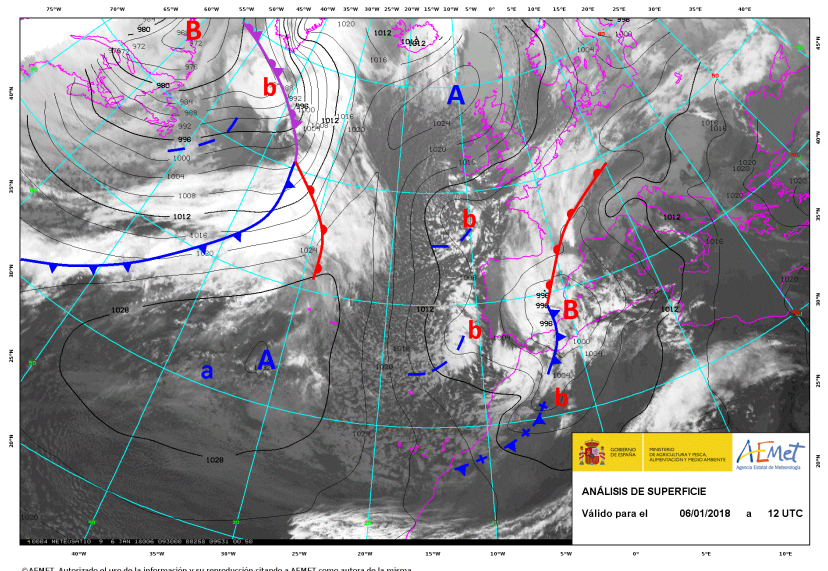

Figura 2. Diagnóstico en superficie a las 12 UTC del día 6.

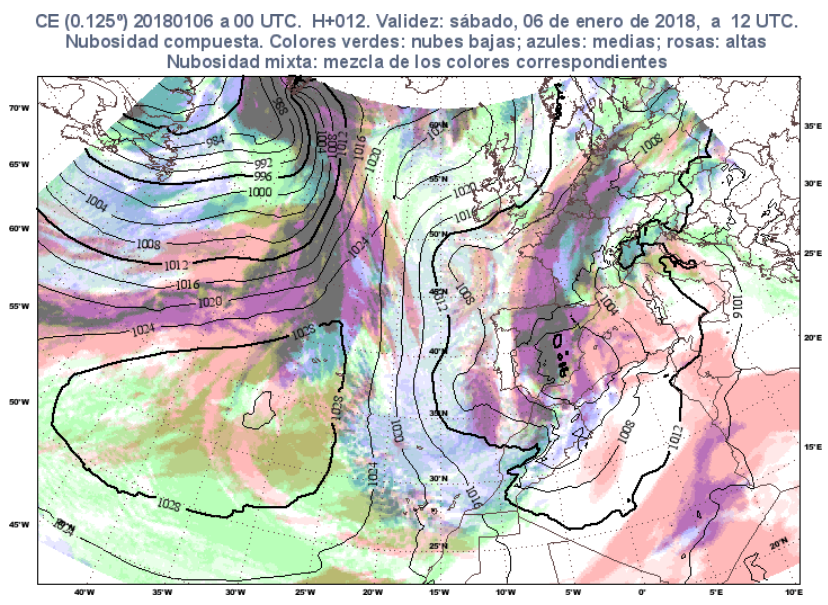

Figura 4. Geopotencial en superficie y nubosidad del ECMWF del día 6 a las 00 UTC previsto para el día 6 a las 12 UTC. 


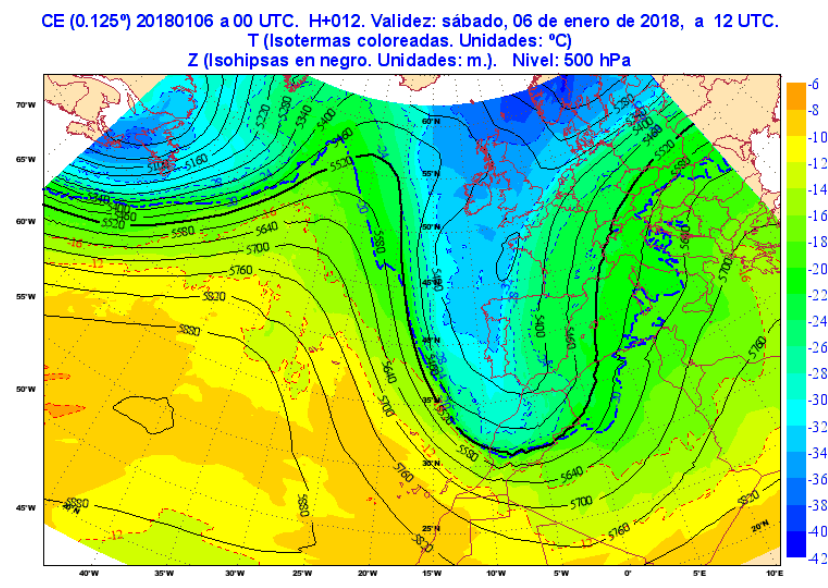

Figura 5. T/Z nivel 500 hPa del ECMWF del día 6 a las 00 UTC previsto para el día 6 a las 12 UTC.

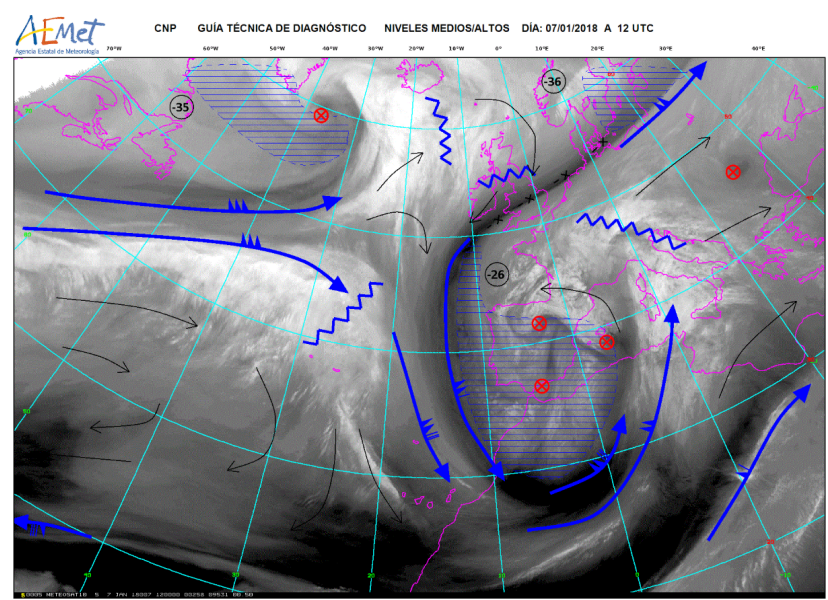

Figura 7. Diagnóstico en niveles medios/altos a las 12 UTC del día 7.

El día 7 prácticamente se repite la situación, como podemos comprobar en las figuras 6 a 9 .

En cuanto a los productos derivados del modelo ECMWF y HARMONIE-AROME, podemos destacar para los días 6 y 7 en la predicción inmediata para las provincias de Ávila y Segovia, lo siguiente:

1. Precipitación en forma de nieve con acumulaciones en 24 horas muy significativas (figura 10 ).

2. EFI (Extreme Forecast Index) extremo, indicando que el episodio de nieve previsto supera a lo climatológicamente conocido (figura 11).

3. Cotas de nieve entre los 500 y $800 \mathrm{~m}$ (figura 12).

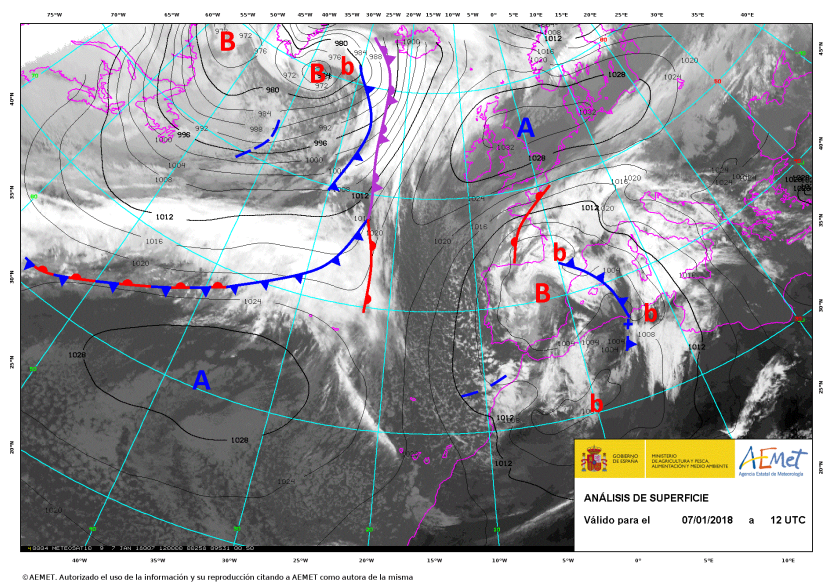

Figura 6. Diagnóstico en superficie a las 12 UTC del día 7.

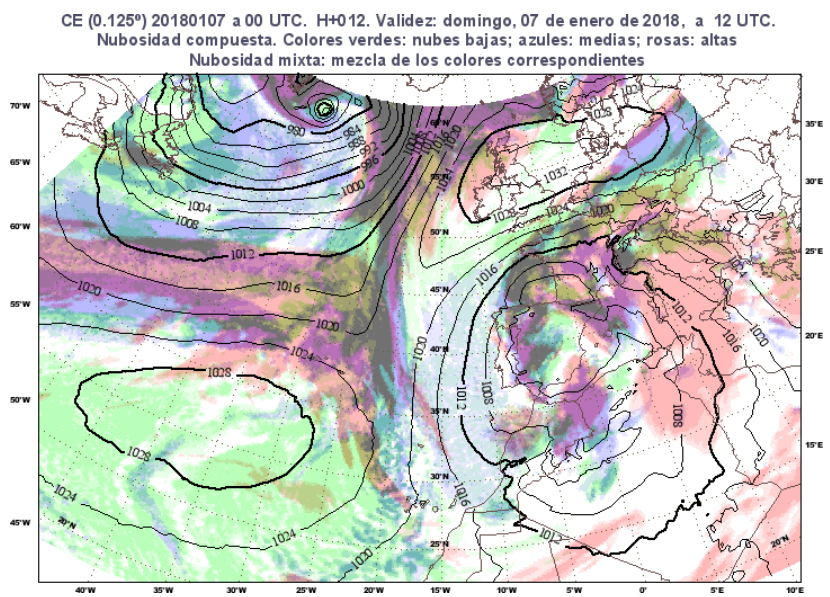

Figura 8. Geopotencial en superficie y nubosidad del ECMWF del día 7 a las 00 UTC previsto para el día 6 a las 12 UTC.

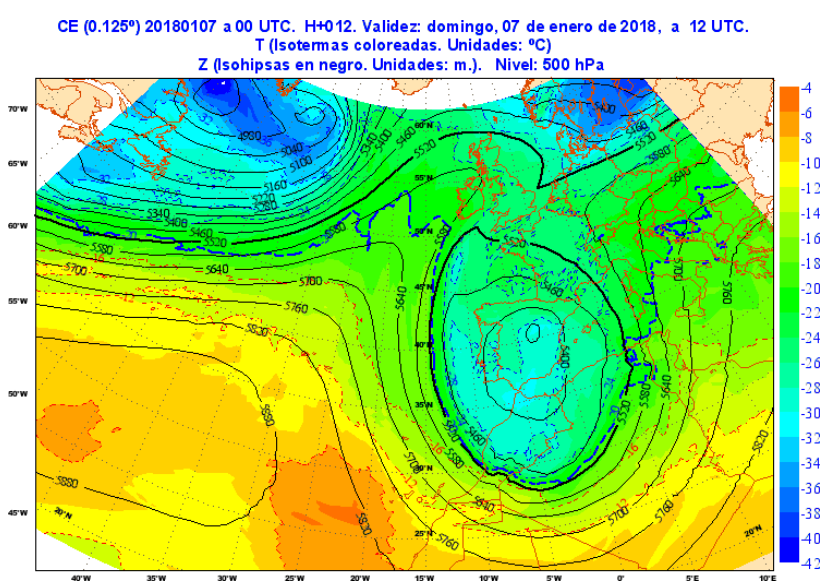

Figura 9. T/Z nivel 500 hPa del ECMWF del día 7 a las 00 UTC previsto para el día 6 a las 12 UTC. 

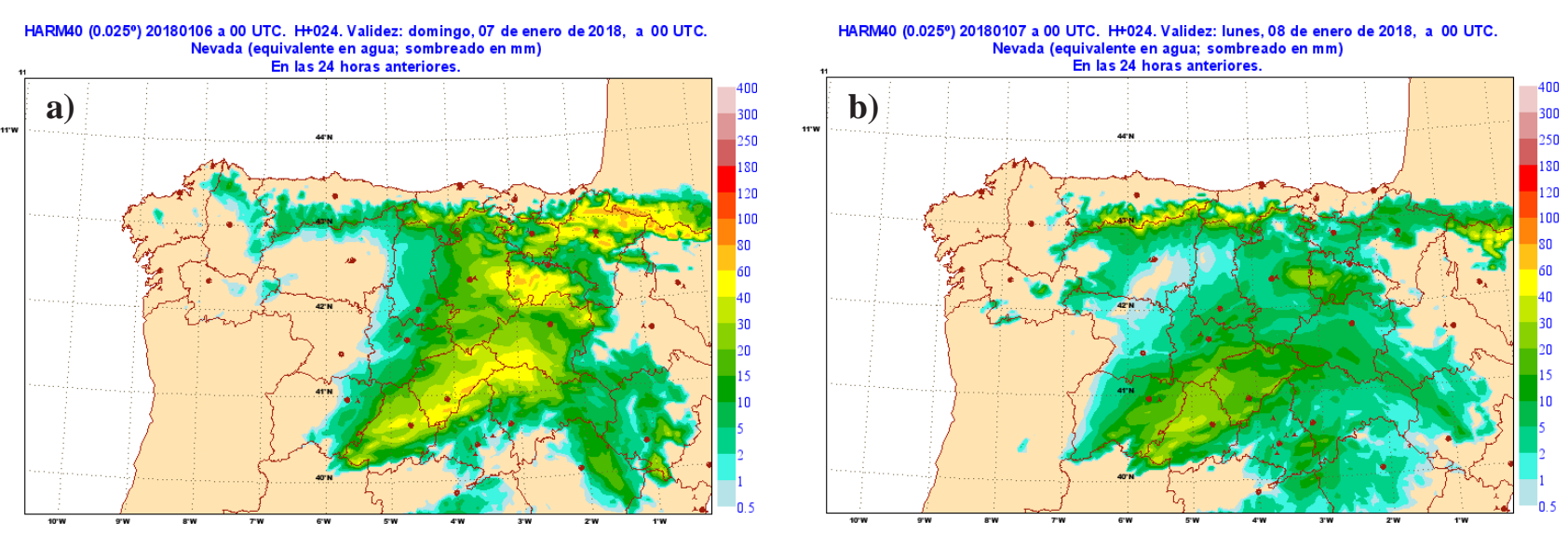

Figura 10. Precipitación en forma de nieve modelo HARMONIE-AROME: a) día 6 y b) día 7.

EPS $\left(0.25^{\circ}\right) 20180106$ a 00 UTC. H+024. Validez: domingo, 07 de enero de 2018, a 00 UTC. EFI nieve acumulada en las $24 \mathrm{~h}$ anteriores( sombreado)
SOT nieve acumulada en las $24 \mathrm{~h}$ anteriores (lineas negras $0,1,5,10,15)$

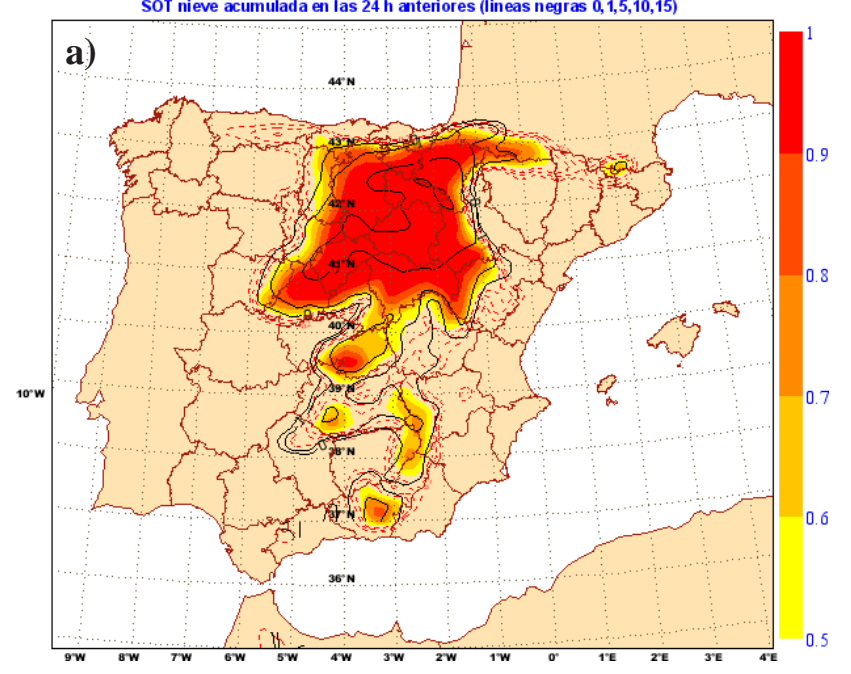

EPS $\left(0.25^{\circ}\right) 20180107$ a 00 UTC. H+024. Validez: lunes, 08 de enero de 2018, a 00 UTC. EFI nieve acumulada en las $24 \mathrm{~h}$ anteriores( (sombreado)
sot nieve acumulada en las $24 \mathrm{~h}$ anteriores (lineas negras $0,1,5,10,15)$

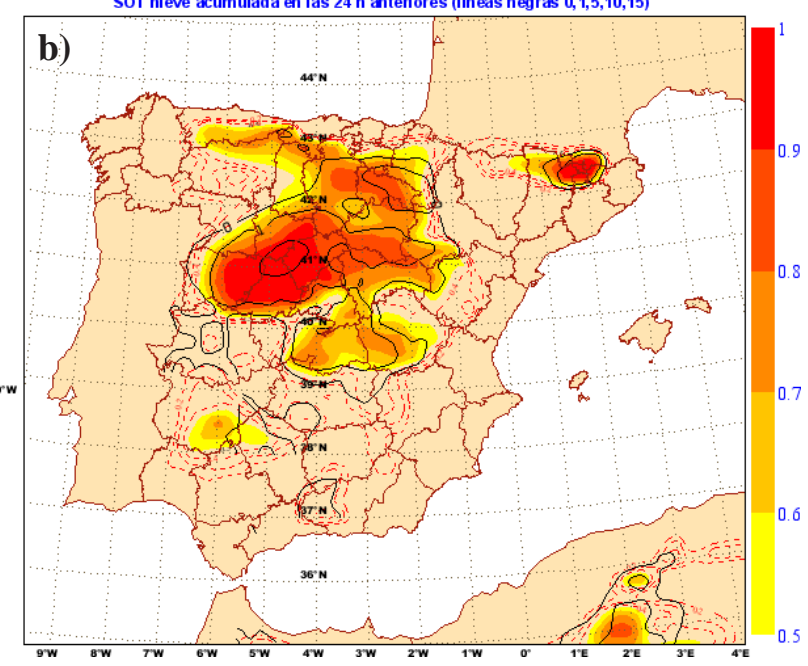

Figura 11. EFI por nieve acumulada: a) día 6 y b) día 7.
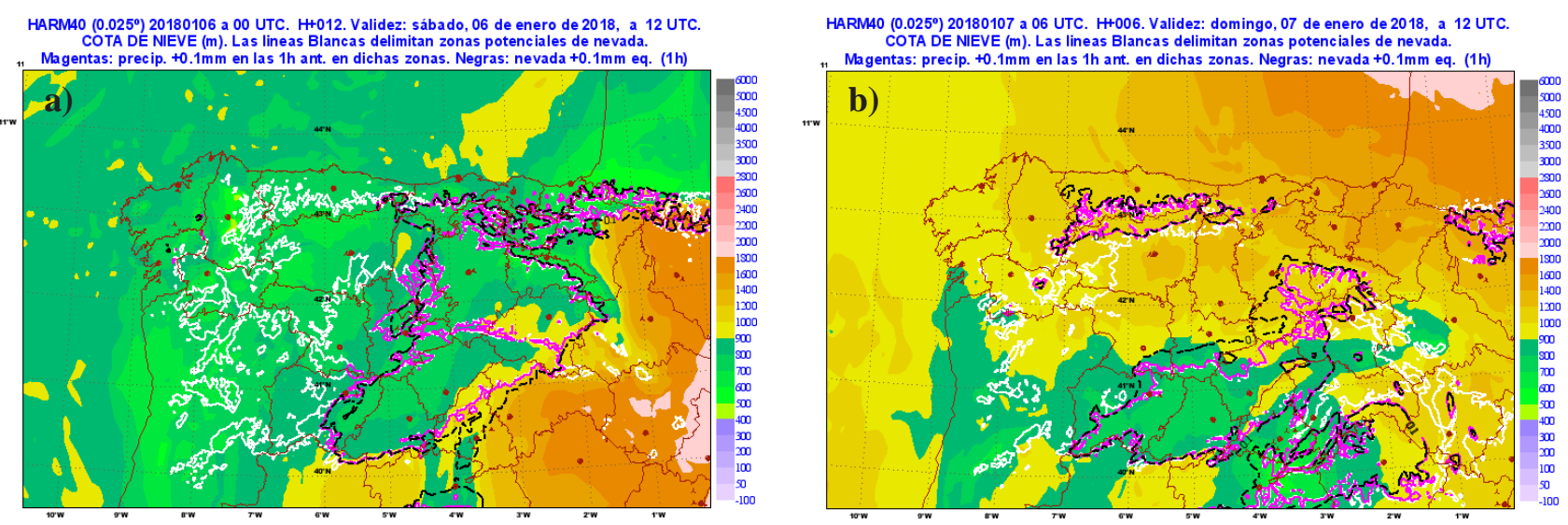

Figura 12. Cota de nieve, modelo HARMONIE-AROME: a) día 6 y b) día 7. 


\subsection{Predicción especial y avisos}

AEMET el 1 de enero, a través de su predicción especial para las fiestas de Navidad, ya pronosticaba este episodio de nieve en el norte peninsular y en cotas bajas.

AEMET el 6 y 7 de enero tenía avisos naranjas por acumulación de nieve en 24 horas en las provincias de Ávila y Segovia tanto en zonas llanas como del sistema Central, salvo en los valles del sur de Ávila.

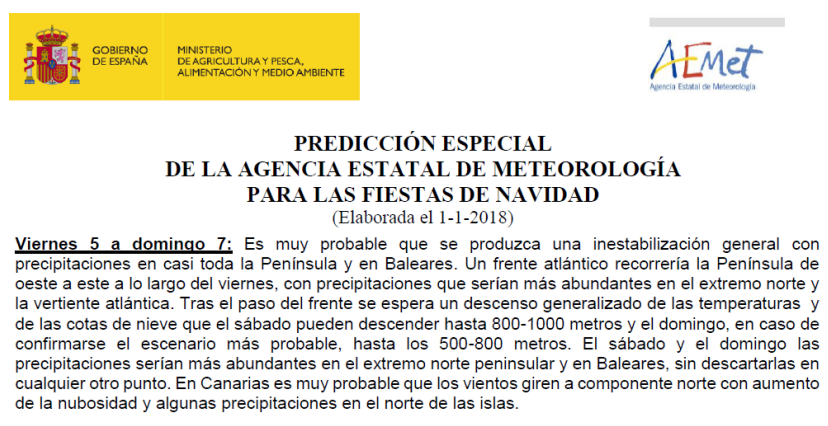

Figura 13. Predicción especial de Navidad elaborada el 1 de enero de 2018.
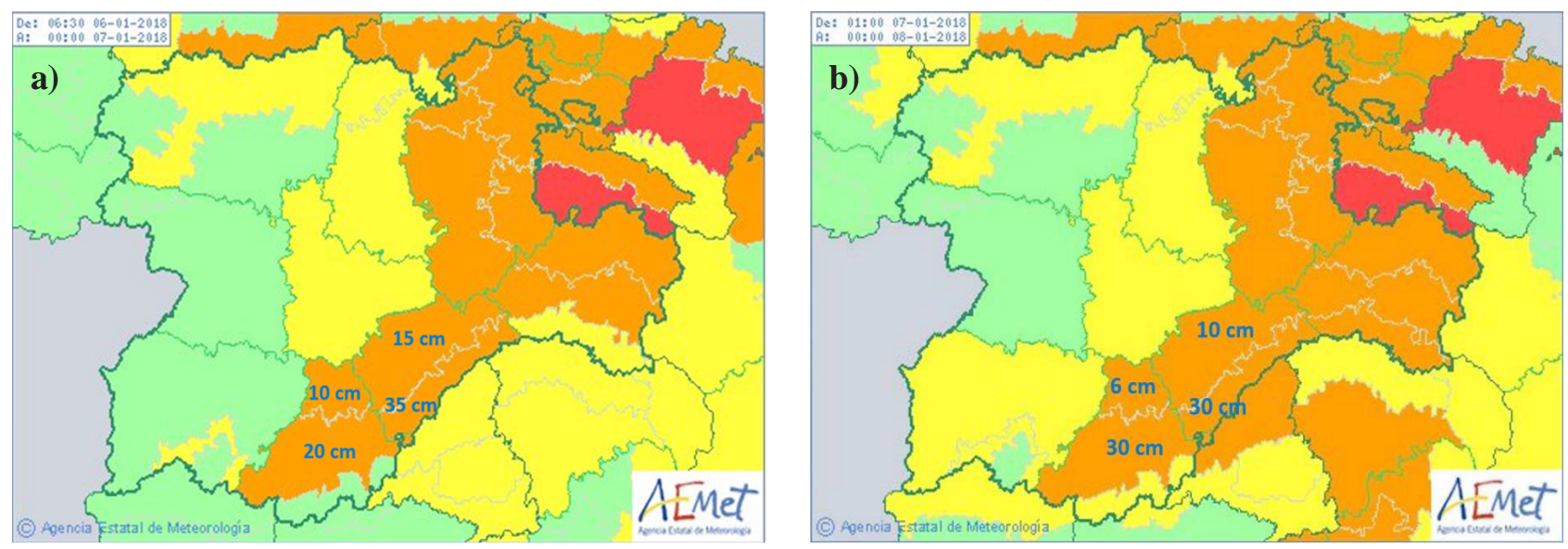

Figura 14. Avisos meteorológicos por acumulación de nieve en 24 horas a) día 6 y b) día 7.

La acumulación de nieve prevista por los avisos en los dos días consecutivos del 6 y 7 de enero dan una cifra muy significativa superando el medio metro en zonas altas y de montaña y entre los 15 y $26 \mathrm{~cm}$ en zonas llanas de la Meseta (véase la tabla1).

\begin{tabular}{|l|c|c|c|}
\cline { 2 - 4 } \multicolumn{1}{c|}{} & Día 6 & Día 7 & Días 6 y 7 \\
\hline Meseta de Segovia & $15 \mathrm{~cm}$ & $10 \mathrm{~cm}$ & $25 \mathrm{~cm}$ \\
\hline Meseta de Ávila & $10 \mathrm{~cm}$ & $6 \mathrm{~cm}$ & $16 \mathrm{~cm}$ \\
\hline Sistema Central Segovia & $35 \mathrm{~cm}$ & $30 \mathrm{~cm}$ & $65 \mathrm{~cm}$ \\
\hline Sistema Central Ávila & $20 \mathrm{~cm}$ & $30 \mathrm{~cm}$ & $50 \mathrm{~cm}$ \\
\hline
\end{tabular}

Tabla 1. Acumulaciones de nieve previstas y por zonas.

Nota: la normativa para avisos meteorológicos adversos (Meteoalerta) para acumulación de nieve solo es sobre 24 horas seguidas.

\section{SITUACIÓN GEOGRÁFICA Y LUGARES DE IMPACTO}

Ávila y Segovia se sitúan en el sur de la Meseta castellana; geográficamente tienen zonas llanas de meseta a la vez que zonas altas y de montaña del sistema Central.

Según el plan Meteoalerta de avisos de fenómenos meteorológicos adversos, las ciudades más pobladas y que a la vez son capitales de provincia, Ávila y Segovia, pertenecen a las zonas de avisos del sistema Central de Ávila y Segovia respectivamente. Ávila se encuentra a 1131 metros de altitud y Segovia a 1002 metros. 
Como lugares de impacto, además de las capitales, las provincias de Ávila y Segovia están cruzadas por autopistas y autovías que unen el nordeste y norte de la Península con Madrid y el sur de España: autopistas AP-6, AP-51, AP-61 y autovía A-1.

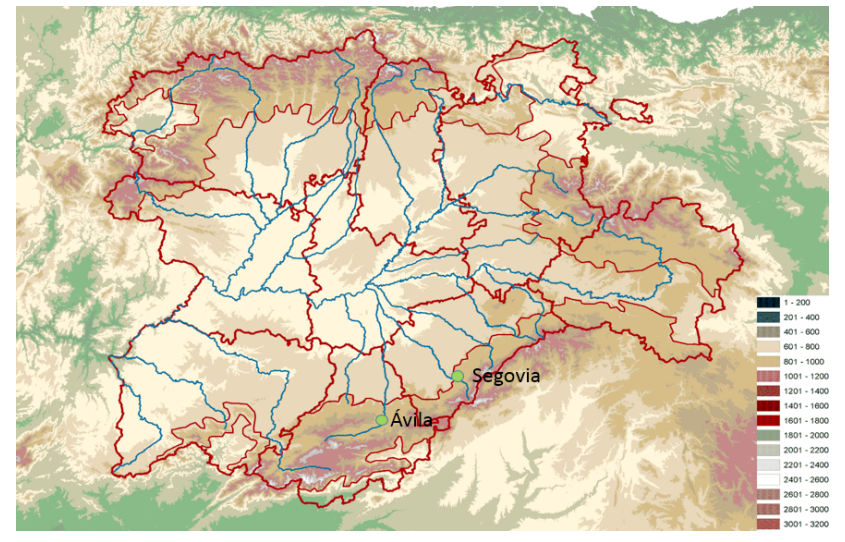

Figura 15. Mapa geográfico.

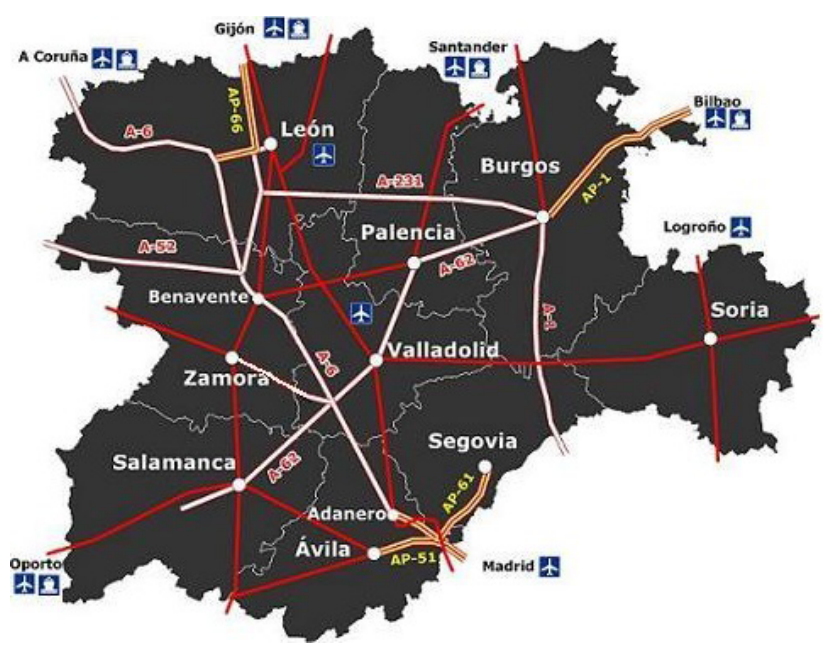

Figura 16. Comunicaciones por carretera.

\section{OBSERVACIÓN}

\subsection{Radar y satélite}

Durante todo el día 6 y las primeras 12 horas del día 7 se observa en las imágenes de satélite la cubierta nubosa y en el radar precipitaciones persistentes en las provincias de Ávila y Segovia. Hay que hacer la indicación de que el radar está situado en Autilla del Pino (Palencia) y los ecos de precipitaciones a bajas altitudes, como es este caso, no las detecta muy bien en esta zona por el enmascaramiento que se tiene por el sistema Central.

El día 8 el cielo estuvo despejado y a través del sensor MODIS del satélite TERRA se pudo observar la gran superficie terrestre con suelo cubierto de nieve (figura 19).

a)

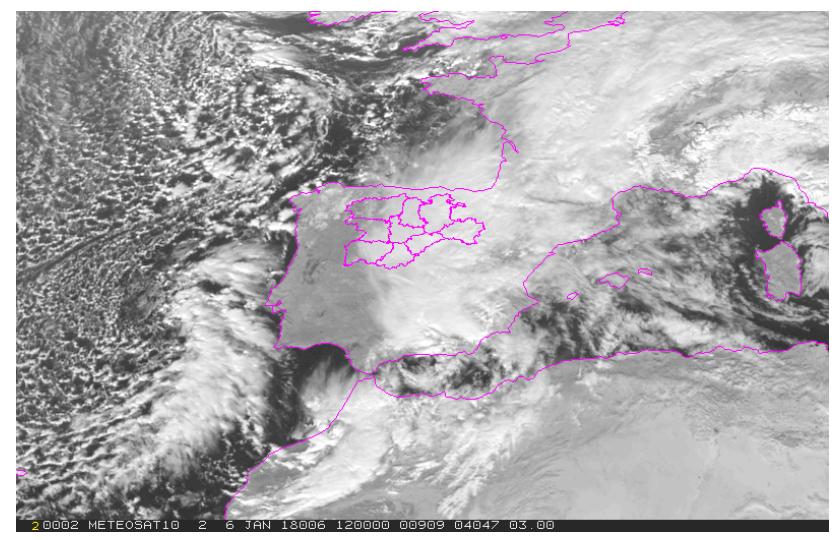

b)

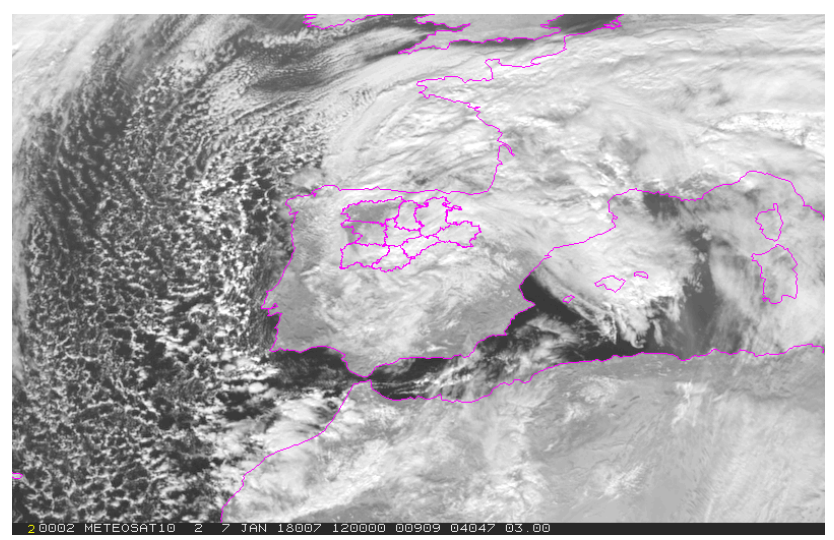

Figura 17. Imagen satélite canal visible a las 12 UTC a) día 6 y b) día 7. 

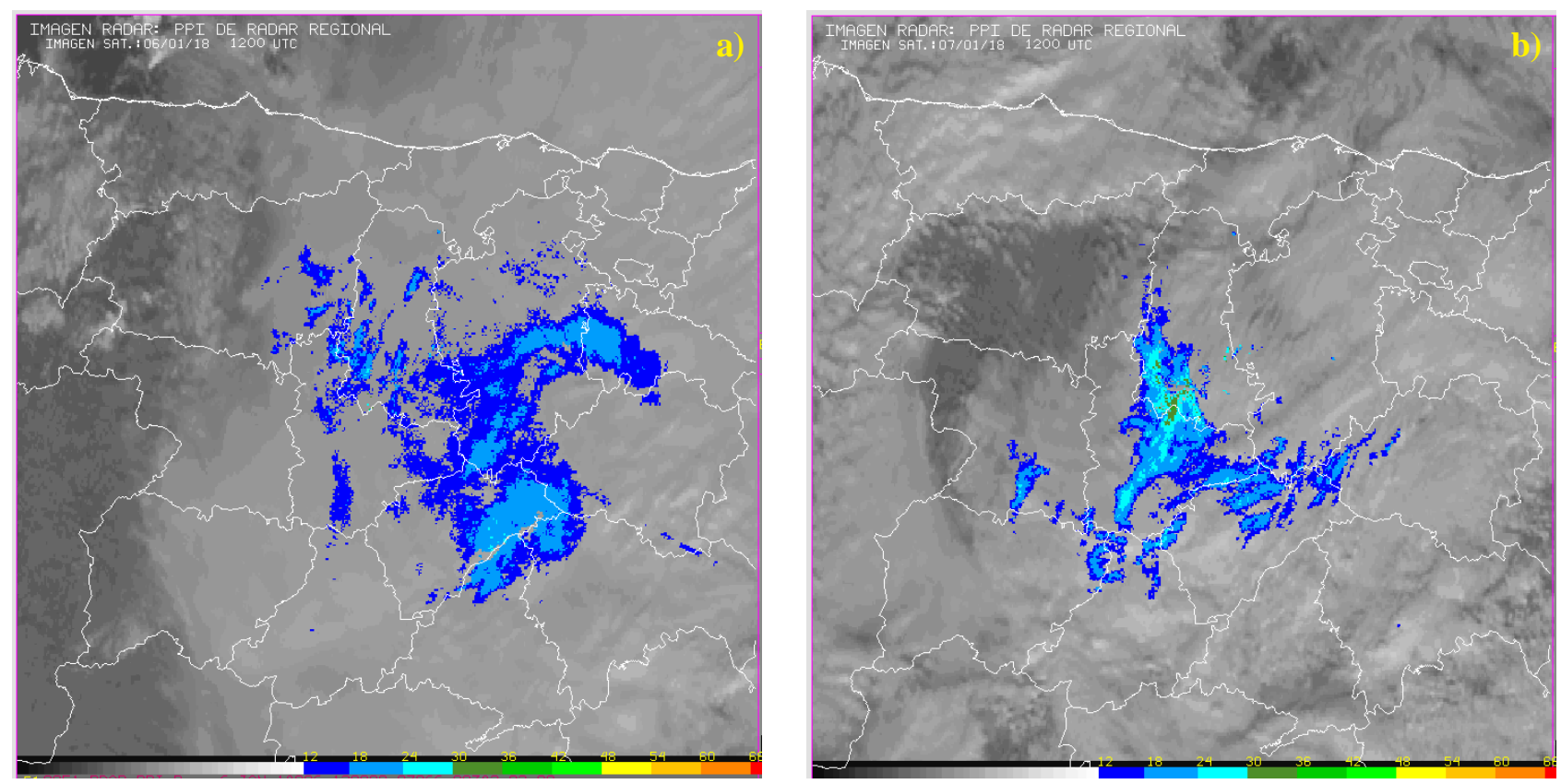

Figura 18. Imagen radar a las 12 UTC a) día 6 y b) día 7.

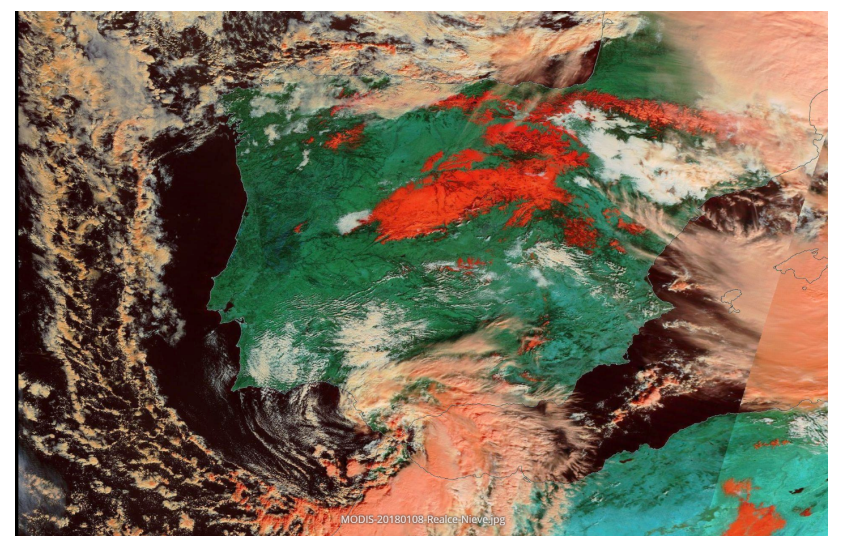

Figura 19.

Imagen realzada MODIS del día 8

(en color rojo la nieve).

\subsection{Superficie}

Se observa que durante los días 6 y 7 en las provincias de Ávila y Segovia la temperatura no superó los 3 grados y en zonas altas, por encima de 900 m, no se pasó de 1 grado como así sucedió en ambas capitales de provincia (figura 20). Esto da idea de que prácticamente toda la precipitación fue en forma de nieve.

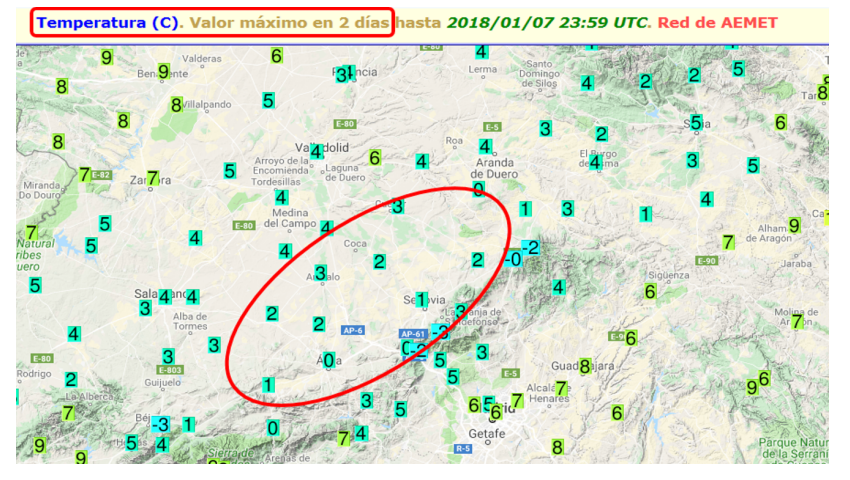

Figura 20. Temperatura máxima en los días 6 y 7 de enero de 2018 en las estaciones automáticas de AEMET.

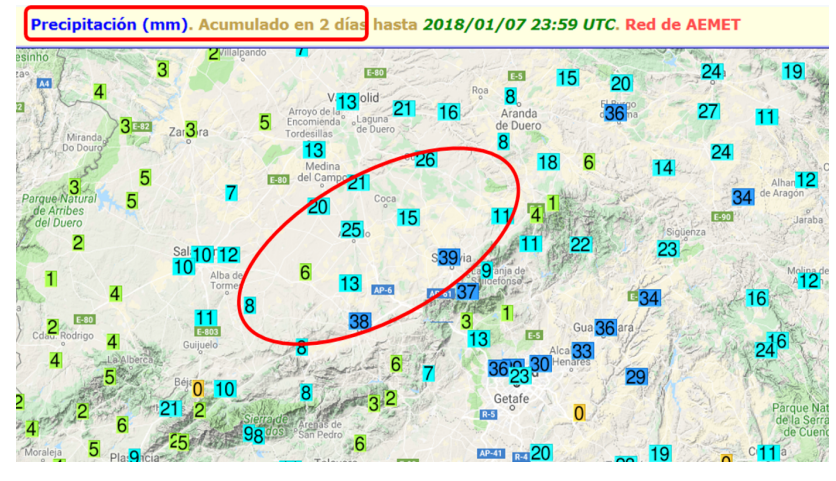

Figura 21. Acumulación de precipitación en los días 6 y 7 de enero de 2018 en las estaciones automáticas de AEMET. 
Se observa la gran cantidad de acumulación de precipitación en las estaciones automáticas durante esos dos días (figura 21).

Tomando como puntos de referencia las localidades de Cuéllar (zona de meseta de Segovia), Arévalo (zona de meseta de Ávila) y los observatorios meteorológicos de las ciudades de Segovia (zona del sistema Central de Segovia) y de Ávila (zona del sistema Central de Ávila) lo previsto se ajusta bastante a lo observado (tabla 2).

\begin{tabular}{|l|c|c|c|c|}
\cline { 2 - 5 } \multicolumn{1}{c|}{} & $\begin{array}{c}\text { Previsto } \\
\text { día } 6\end{array}$ & $\begin{array}{c}\text { Previsto } \\
\text { día } 7\end{array}$ & $\begin{array}{c}\text { Previsto } \\
\text { días 6 y } 7\end{array}$ & $\begin{array}{c}\text { Observado } \\
\text { días 6 y } 7\end{array}$ \\
\hline Meseta de Segovia & $15 \mathrm{~cm}$ & $10 \mathrm{~cm}$ & $25 \mathrm{~cm}$ & $\begin{array}{c}\text { Cuéllar: } \\
25,8 \text { litros/m² en pluviómetro }\end{array}$ \\
\hline Meseta de Ávila & $10 \mathrm{~cm}$ & $6 \mathrm{~cm}$ & $16 \mathrm{~cm}$ & $\begin{array}{c}\text { Arévalo: } \\
25,0 \text { litros/m² } / \mathrm{m}^{2} \text { pluviómetro }\end{array}$ \\
\hline Sistema Central Segovia & $35 \mathrm{~cm}$ & $30 \mathrm{~cm}$ & $65 \mathrm{~cm}$ & $\begin{array}{c}\text { Observatorio Segovia: } \\
45 \mathrm{~cm} \text { en suelo }\end{array}$ \\
\hline Sistema Central Ávila & $20 \mathrm{~cm}$ & $30 \mathrm{~cm}$ & $50 \mathrm{~cm}$ & $\begin{array}{c}\text { Observatorio Ávila: } \\
44 \mathrm{~cm} \text { en suelo }\end{array}$ \\
\hline
\end{tabular}

Tabla 2. Acumulaciones de nieve previstas y observadas por zonas.

\section{PROTOCOLOS DE PROTECCIÓN CIVIL EN LA DELEGACIÓN DE GOBIERNO EN CASTILLA Y LEÓN}

En las figuras 22 a 25 se puede ver el seguimiento de la Delegación del Gobierno en Castilla y León de este episodio de nevada a través de su twitter oficial (@DGCyL).

Se observa que se aplicaron los protocolos de alerta el día 5 y de emergencia el día 6.

Hay que recordar que AEMET emite avisos por fenómenos meteorológicos adversos, y son las instituciones, a través de sus protocolos de protección civil, las que emiten alertas y emergencias.

Delegación Gob CyL

$@ D G C y L$

Cambia el \#tiempo y recibimos a los \#ReyesMagos2018 con \#precipitaciones en forma de \#nieve y descenso de los termómetros. Planifica tu viaje y consulta \#avisos y predicción vía @AEMET_Esp @AEMET_CyL

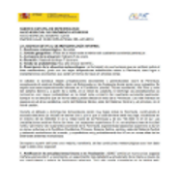

AEMET @AEMET_Esp

\#AvisoEspecial por \#nevadas .... para el sábado y el domingo aemet.es/documentos_d/e.... Muy atentos $\bullet$ a los detalles $y$ actualizaciones en aemet.es/es/eltiempo/pr...

20:07 - 4 ene. 2018 desde Castilla y León, España

Figura 22. Twitt de @DGCyL del día 4 de enero de 2018.
Delegación Gob CyL

$@ D G C y L$

Siguiendo

La Delegación del Gobierno en \#CastillayLeón ha activado la fase de alerta por \#nevadas

*⿻丷木 @DGCyL recuerda las medidas de prevención y autoprotección que se deben seguir ante temporales de nieve y frío en viajes y estancias en zonas afectadas.
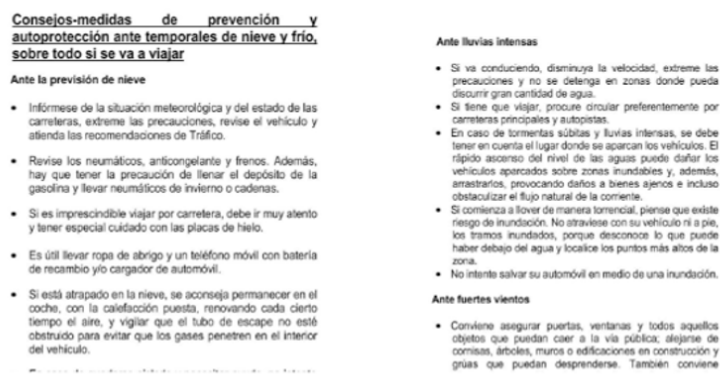

14:51 - 5 ene. 2018 desde Castilla y León, España

Figura 23. Twitt de @DGCyL del día 5 de enero de 2018 de activación de fase de alerta por nevadas. 
Delegación Gob CyL

@DGCyL

@DGCyL ha establecido la declaración de la fase de emergencia del protocolo de coordinación de actuaciones ante situaciones meteorológicas extremas, que afectan a la Red de \#carreteras del Estado en las provincias de \#Segovia y \#Ávila desde la 20h y hasta nuevo aviso

21:29 - 6 ene. 2018

Figura 24. Twitt de @DGCyL del día 6 de enero de 2018 de activación de fase de emergencia por nevadas.

\section{Delegación Gob CyL}

$@ D G C y L$

Se establece la desactivación de la fase de Alerta de emergencia del Protocolo de coordinación por nevadas en la Red de \#carreteras del Estado en todas las provincias de \#CastillayLeón desde las $11.00 \mathrm{~h}$. @DGCyL

13:08 - 8 ene. 2018 desde Valladolid, España

Figura 25. Twitt de @DGCyL del día 8 de enero de 2018 de desactivación de la fase de emergencia por nevadas.

\section{IMPACTOS}

El mayor impacto se produjo en la red de comunicaciones por carretera, y especialmente en la autopista AP-6 que une el noroeste de España con Madrid en la provincia de Segovia. Más de 3000 vehículos quedaron atrapados por la nieve durante la noche del 6 al 7 de enero de 2018.

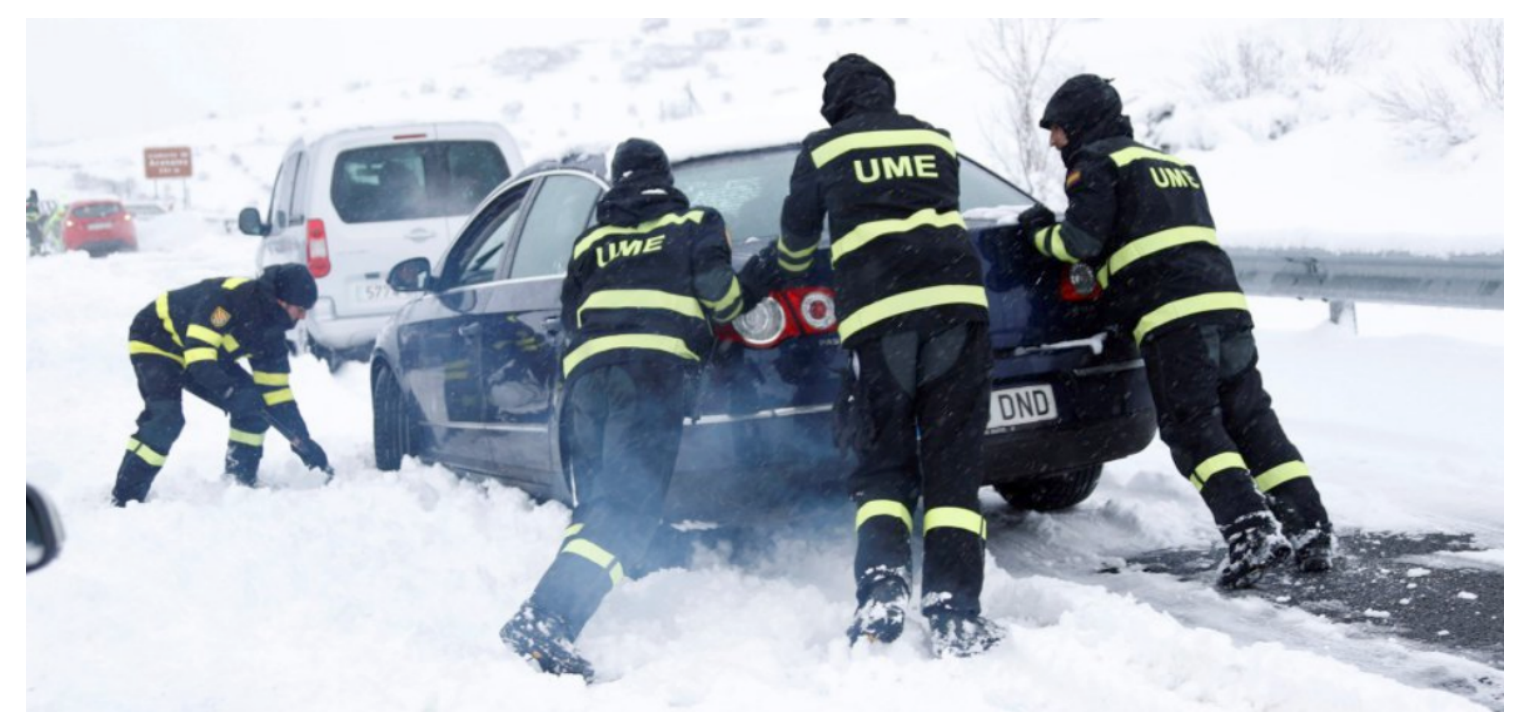

Figura 26. Intervención de la Unidad Militar de Emergencias (UME) en la autopista AP-6 ayudando en el rescate de coches atrapados por la nieve.

\section{CONCLUSIONES}

Este episodio de nieve estuvo muy bien previsto tanto por los modelos numéricos de predicción, como por los predictores operativos en sus predicciones y avisos.

Si las predicciones no hubiesen sido tan acertadas, el impacto que podía haber tenido este episodio de nieve podría haber sido mayor.

Para haber minimizado al máximo el impacto de este episodio es probable que haya que ir mejorando los avisos de fenómenos meteorológicos adversos orientándolos más a los impactos concretos que puedan originar. 


\section{AGRADECIMIENTOS}

Este trabajo ha sido posible gracias a la colaboración del Área de Técnicas y Aplicaciones de Predicción (ATAP), al Sistema Nacional de Predicción y a los observatorios meteorológicos en las ciudades de Ávila y Segovia. Todos ellos pertenecen a AEMET.

\section{REFERENCIAS}

http://www.aemet.es/documentos/es/eltiempo/prediccion/avisos/plan_meteoalerta/plan_meteoalerta.pdf. 